\title{
HÉRNIA DISCAL LOMBAR
}

\author{
LUMBAR DISC HERNIATION
}

Luis Roberto Vialle', Emiliano Neves Vialle², Juan Esteban Suárez Henao ${ }^{3}$, Gustavo Giraldo³

\section{RESUMO}

A hérnia discal lombar é o diagnóstico mais comum dentre as alterações degenerativas da coluna lombar (acomete 2 a 3\% da população) e a principal causa de cirurgia de coluna na população adulta. O quadro clínico típico inclui lombalgia inicial, seguida de lombociatalgia e, finalmente, de dor ciática pura. A história natural da hérnia de disco é de resolução rápida dos sintomas (quatro a seis semanas). O tratamento inicial deve ser conservador, com manejo medicamentoso e fisioterápico, podendo ser acompanhado ou não por bloqueios percutâneos radiculares. O tratamento cirúrgico está indicado na falha do controle da dor, déficit motor maior que grau 3, dor radicular associada à estenose óssea foraminal ou síndrome de cauda equina, sendo esta última uma emergência médica. Uma técnica cirúrgica refinada, com remoção do fragmento extruso, e preservação do ligamento amarelo, resolve a sintomatologia da ciática e reduz a possibilidade de recidiva em longo prazo.

Descritores - Hérnia discal lombar; Ligamento amarelo; Ciática; Tratamento cirúrgico

\section{INTRODUÇÃO}

A hérnia discal lombar consiste de um deslocamento do conteúdo do disco intervertebral - o núcleo pulposo - através de sua membrana externa, o ânulo fibroso, geralmente em sua região posterolateral. Dependendo do volume de material herniado, poderá haver compressão e irritação das raízes lombares e do saco dural, representadas clinicamente pela dor conhecida como ciática. Essa dor é conhecida desde a Antiguidade, mas a sua relação com a hérnia discal não foi descoberta até o início do século 20, quando Mixter e Barr a descreveram ${ }^{(1)}$.

Atualmente, a hérnia discal lombar é o diagnóstico mais comum dentre as alterações degenerativas da co-

\section{ABSTRACT}

Lumbar disc herniation is the most common diagnosis amongst the degenerative conditions of the lumbar spine (affecting around 2 to $3 \%$ of the population), and is the principal cause of spine surgery in the adult population. The typical clinical picture includes initial lumbalgia, followed by progressive sciatica. The natural history of disc herniation is one of rapid resolution of the symptoms (from 4-6 weeks). Early treatment should be conservative, with pain management and physiotherapy, sometimes associated with selective nerve root block. Surgery should be considered if pain management is unsuccessful, if there is a motor deficit (strength grade 3 or less), where there is radicular pain associated with foraminal stenosis, or in the presence of cauda equina syndrome, the latter representing a medical emergency. A refined surgical technique, with removal of the extruded fragment and preservation of the ligamentum flavum, resolves the sciatic symptoms and reduces the risk of recurrence in the long term.

Keywords - Lumbar disc herniation; Ligamentum flavum; Sciatica; Surgical treatment

luna lombar e a principal causa de cirurgia de coluna. Fatores como maior acesso a cuidados médicos, precocidade na solicitação de exames de imagem e segurança dos procedimentos cirúrgicos levam a altas taxas de cirurgia, condição que é geralmente autolimitada.

O tratamento da hérnia discal lombar evoluiu das ressecções transdurais para a abordagem convencional ${ }^{(2,3)}$, seguida da microcirurgia até a cirurgia endoscópica e percutânea. Atualmente. a hérnia discal lombar é a condição que mais leva à cirurgia de coluna, principalmente em homens ao redor dos 40 anos de idade ${ }^{(4)}$.

Esta revisão visa discutir os principais aspectos de epidemiologia, diagnóstico e tratamento da hérnia discal lombar.

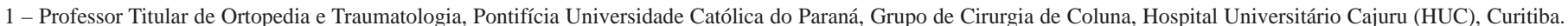

2 - Grupo de Cirurgia de Coluna, Hospital Universitário Cajuru, Pontifícia Universidade Católica do Paraná.

3 - Estagiário em Cirurgia de Coluna, Hospital Universitário Cajuru, Pontifícia Universidade Católica do Paraná.

Correspondência: Rua Brigadeiro Franco, 979 - 80430-210 - Curitiba, PR. E-mail: vialle@uol.com.br 


\section{EPIDEMIOLOGIA}

A hérnia discal ocorre principalmente entre a quarta e quinta décadas de vida (idade média de 37 anos), apesar de ser descrita em todas as faixas etárias ${ }^{(5-8)}$. Estima-se que 2 a 3\% da população possam ser afetados, com prevalência de 4,8\% em homens e 2,5\% em mulheres, acima de 35 anos.

Por ser tão comum, chega a ser considerada um problema de saúde mundial, em decorrência de incapacidade que gera ${ }^{(9)}$.

Apesar de se atribuir ao tabagismo, exposição a cargas repetidas e vibração prolongada um risco aumentado de hérnia discal, estudos mostram que a diferença é pequena, quando a população exposta a esses fatores é comparada com grupos controle ${ }^{(10,11)}$.

A predisposição genética tem sido alvo de estudos recentes, envolvendo genes como o receptor da vitamina D, $\mathrm{VDR}^{(12,13)}$, o gene que codifica uma das cadeias polipeptídicas do colágeno IX, COL9A2 ${ }^{(14)}$ e o gene aggrecan humano (AGC), responsável pela codificação do proteoglicano, maior componente proteico da cartilagem estrutural, que suporta a função biomecânica nesse tecido ${ }^{(15-17)}$.

\section{PATOGÊNESE DA CIÁTICA}

A origem da dor ciática é provavelmente multifatorial, envolvendo estímulo mecânico das terminações nervosas da porção externa do ânulo fibroso, compressão direta da raiz nervosa (com ou sem isquemia) e uma série de fenômenos inflamatórios induzidos pelo núcleo extruso ${ }^{(18)}$.

O fator que desencadeia a dor ciática é a compressão mecânica da raiz nervosa pela hérnia discal. Em decorrência desta, há isquemia e fenômenos que sensibilizam a membrana à dor ${ }^{(19,20)}$. Estudos demonstram que o limiar de sensibilização neuronal para uma raiz comprimida é cerca da metade do dos segmentos não comprimidos $^{(21,22)}$.

Existe uma diferença nos níveis de infiltração celular inflamatória em hérnias discais extrusas e não extrusas, menores nestas últimas ${ }^{(6,7)}$. Acredita-se que a ruptura do ligamento longitudinal posterior pelas hérnias extrusas as expõe ao leito vascular do espaço epidural, e células inflamatórias originadas desses vasos na periferia do material discal herniado podem ter um papel importante na irritação da raiz nervosa e na indução da dor ciática. Isso pode explicar por que hérnias extrusas têm maior compromisso clínico e maior frequência de reabsorção ${ }^{(7)}$. Portanto, pode-se afirmar que em pacientes com hérnias contidas predomina o efeito compressivo mecânico e em pacientes com hérnias não contidas - extrusas - predomina o componente inflamatório.

\section{Quadro clínico}

O quadro clínico típico de uma hérnia discal inclui lombalgia inicial, que pode evoluir para lombociatalgia (em geral, após uma semana) e, finalmente, persistir como ciática pura. Mas devido às inúmeras possibilidades de apresentação de formas agudas ou crônicas, deve-se estar atento a formas atípicas de apresentação e preparado para fazer um apurado diagnóstico diferencial. Apesar de ser a principal causa de dor ciática, outras possibilidades, tais como tumores, instabilidade, infecção, devem ser afastadas. Um exame físico adequado é essencial para isso, podendo inclusive, através de cuidadosa avaliação de dermátomos e miótomos, determinar o espaço vertebral em que está localizada a hérnia.

O que é importante enfatizar é que a história natural da ciática por hérnia de disco é de resolução acentuada dos sintomas em torno de quatro a seis semanas. Por essa razão é que o tratamento inicial deve ser sempre conservador, explicando ao paciente que o processo tem um curso favorável.

\section{Diagnóstico por imagem}

A radiografia, por ser rotineira e de baixo custo, deve fazer parte da avaliação por imagem. Embora o quadro clínico possa ser claro e sugestivo de hérnia discal, não se deve esquecer da possibilidade de coexistirem outras alterações que podem ser detectadas pela radiografia. O exame ortostático e o dinâmico em flexão e extensão são complementações importantes para análise mais completa da coluna.

O exame de eleição é a ressonância magnética (RM). No Brasil ainda se insiste no uso da tomografia axial computadorizada que, apesar de ser capaz de identificar uma hérnia de disco, não se aproxima da qualidade e tampouco da sensibilidade da RM. Informações detalhadas de partes ósseas e de tecidos moles que podem ajudar não só no diagnostico correto, mas até na proposta terapêutica, fazem da RM o exame indispensável para a correta avaliação do paciente.

Na RM, a hérnia é classificada segundo sua forma. Essa descrição morfológica é a seguinte, de forma resumida. O material discal, proveniente principalmente do núcleo pulposo, está deslocado para fora dos limites intervertebrais, podendo tomar três formas diferentes: protrusão, extrusão ou sequestro.

Protrusão é quando a distância da altura da hérnia (no corte axial) é menor que a distância da base em qualquer um dos planos (Figura 1). Extrusão é quando a distância da base é menor que a altura da hérnia (Figura 2), e sequestro, quando não há continuidade entre o material herniado e o disco intervertebral ${ }^{(23)}$ (Figura 3). 


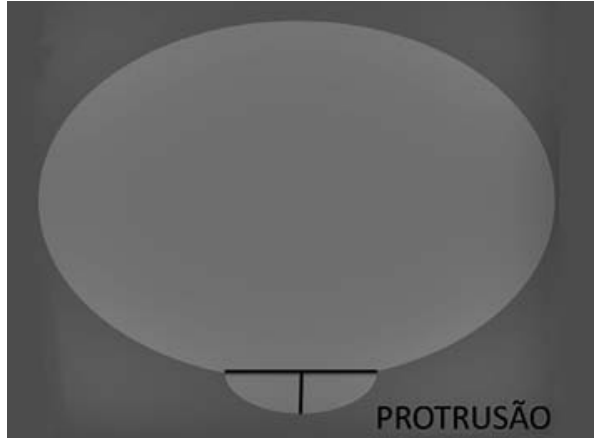

Figura 1 - Protrusão: base maior que altura

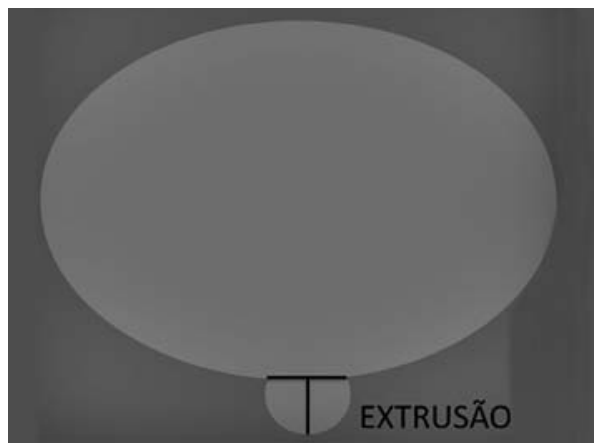

Figura 2 - Extrusão: altura maior que base

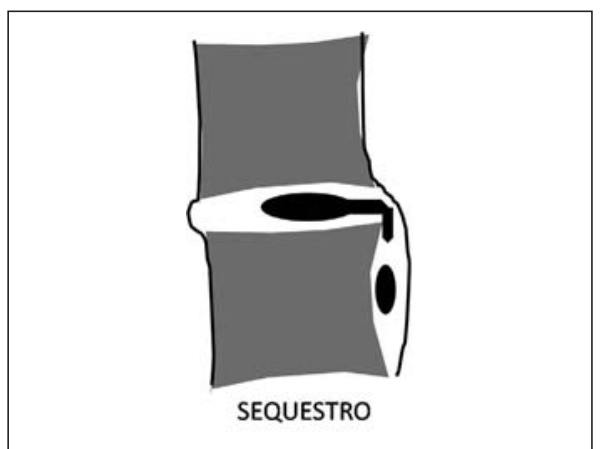

Figura 3 - Sequestro: sem continuidade com o disco

A protrusão pode ser focal, interpretada como protrusão focal, ou ampla e concêntrica, chamada de protrusão concêntrica (bulging em inglês, termo usado em alguns laudos) (Figura 4).

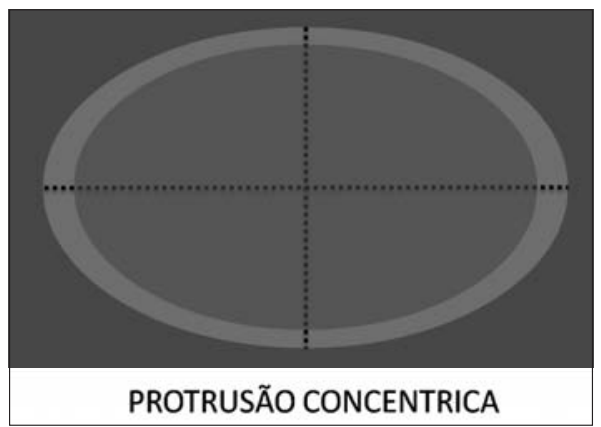

Figura 4 - Protrusão focal e concêntrica

\section{TRATAMENTO}

\section{Tratamento conservador}

A hérnia de disco lombar é uma condição que apresenta natureza benigna; a finalidade do tratamento é aliviar a dor, estimular a recuperação neurológica, com retorno precoce às atividades da vida diária e ao trabalho. Pacientes com hérnias sequestradas, jovens, com leve déficit neurológico, hérnias pequenas, pouca degeneração discal, seriam os que melhor se beneficiariam do tratamento conservador ${ }^{(24)}$. A crise de ciática pode ser tão severa a ponto de incapacitar o paciente e, nesse momento, o tratamento deve reduzir gradativamente a dor e aumentar a atividade física, evitando o repouso absoluto. Os AINES são os medicamentos que mais devem ser utilizados, por atender exatamente às necessidades da fisiopatologia - basicamente inflamatória - ficando os analgésicos puros como terapia adicional.

Como já foi mencionado, a historia natural da ciática se caracteriza por um rápido alívio da sintomatologia num tempo médio de quatro a seis semanas, com recorrência de aproximadamente 5 a 10\%, sem importar o tipo de tratamento instaurado ${ }^{(25)}$.

Uma alternativa para ajudar o tratamento conservador é o bloqueio da raiz afetada com anestésico e corticoide, que atua diretamente sobre a hérnia, reduzindo seu volume, e sobre a raiz, reduzindo a sua resposta inflamatória ${ }^{(26-28)}$. Num estudo realizado pelo Grupo de Cirurgia da Coluna do Hospital Universitário Cajuru (HUC), foram avaliados 70 pacientes portadores de hérnia discal lombar com radiculopatia, com quadro de dor por até oito semanas; após o $3 .^{\circ}$ mês do bloqueio, 77\% dos pacientes estavam assintomáticos. Foi possível concluir que o bloqueio anestésico transforaminal é uma alternativa eficaz e segura no tratamento da dor ciática secundaria à hérnia de disco lombar ${ }^{(29)}$.

Buenaventura et al, numa revisão sistemática da literatura sobre bloqueio transforaminal lombar, encontraram evidência nível II-1 para alívio em curto prazo (seis meses ou menos) e nível II-2 para o alívio em longo prazo (mais de seis meses) no tratamento da dor ciática ${ }^{(30)}$. Nossa conduta preferencial hoje é iniciar o tratamento da dor aguda com esse tipo de bloqueio.

O tratamento conservador inclui fisioterapia de apoio com analgesia e relaxamento, principalmente através de exercícios e alongamentos. O uso de 
estimulação elétrica nas suas formas mais variadas (TENS) não apresenta nenhuma evidência que justifique sua utilização; os estudos publicados mostram que não há fundamentos importantes estabelecendo o seu valor ${ }^{(31)}$.

\section{Tratamento cirúrgico}

O objeto do tratamento cirúrgico é a descompressão das estruturas nervosas. As indicações do tratamento cirúrgico são:

Absolutas: Síndrome de cauda equina ou paresia importante.

Relativas: Ciática que não responde ao tratamento conservador pelo menos por seis semanas, déficit motor maior que grau 3 e ciática por mais de seis semanas ou dor radicular associada à estenose óssea foraminal.

Nos últimos anos tem sido muito discutida a vantagem entre cirurgia precoce e tratamento conservador prolongado. Há publicações mostrando resultados clínicos similares nos grupos estudados, após dois anos de evolução, mas a recuperação foi mais rápida no grupo da cirurgia precoce. Os autores mostraram que é economicamente favorável o tratamento cirúrgico, por permitir retorno precoce ao trabalho ${ }^{(32,33)}$.

\section{TÉCNICAS CIRÚRGICAS}

Embora a discectomia tradicional ainda seja a técnica usada por alguns cirurgiões, operações minimamente invasivas vêm ganhando atenção nos últimos anos e a microdiscectomia pode ser um meio-termo entre os dois $\operatorname{polos}^{(34)}$.

Duas abordagens cirúrgicas têm sido propostas. A cirurgia tradicional, conhecida como "laminectomia", não tem mais lugar. $\mathrm{O}$ que se estuda hoje em dia é a vantagem de procedimentos minimamente invasivos ou percutâneos sobre a microdiscectomia. Os resultados favoráveis da microdiscectomia tanto em curto prazo - tempo cirúrgico, sangramento, alívio dos sintomas, taxa de complicações - quanto aos 10 anos de seguimento ainda fazem com que essa seja a técnica preferencial. Recentemente, foram publicados alguns estudos comparando as duas técnicas, sem conseguir estabelecer diferenças importantes ${ }^{(35)}$. Em um estudo randomizado que analisou durante dois anos os dois procedimentos, houve resultado favorável à microdiscectomia $^{(36)}$.

Também se discute a remoção extensa dos fragmentos de disco e curetagem do espaço discal, ou apenas a retirada do fragmento herniado com mínima invasão ao espaço $\operatorname{discal}^{(37,38)}$.

Watters e McGirt ${ }^{(35)}$ encontraram evidência a favor da remoção apenas do fragmento herniado considerando a duração da cirurgia e o regresso à atividade laboral.

Ao comparar as duas táticas, encontra-se um aumento significativo na incidência de dores lombares quando é realizada a remoção agressiva do disco, em oposição à técnica conservadora (28\% x 11,5\%). Estudos biomecânicos demonstram que o aumento da lesão do espaço discal acelera a doença degenerativa. A quantidade de disco retirado durante o procedimento cirúrgico estaria associada a piores resultados clínicos em longo prazo, no que diz respeito ao aparecimento de lombalgia. Mas a recorrência da hérnia de disco, com taxa média em torno de $7 \%$, é maior quando se utiliza a técnica conservadora $^{(39-41)}$.

Isso tem impacto, especialmente econômico, quando se decide por um complemento cirúrgico adicional, artrodese ou artroplastia. Bastante discutível, só se aplicaria em discos jovens e com altura ainda normal, onde, em tese, poderia ocorrer instabilidade. Casos bem selecionados, com história de lombalgia prévia importante e discos altos poderiam beneficiar-se desses procedimentos. Mas deve ficar claro que a artrodese ou a artroplastia não têm lugar no tratamento da hérnia de disco convencional.

\section{Preservação do ligamento amarelo}

Após discectomia lombar há um processo de cicatrização perirradicular com acúmulo de material fibroso substituindo a gordura peridural. Essa gordura tem menor conteúdo de colágeno que a gordura subcutânea ${ }^{(42)}$, o que permite que as raízes e o saco dural se mobilizem livremente no espaço peridural sem compressão nem aderências. A fibrose peridural pode fixar as raízes e a dura-máter aos tecidos circundantes, comprometendo a nutrição e a atividade dinâmica do segmento; a alteração de fluxos arteriais e venosos de estruturas sensíveis à deformação mecânica, como o gânglio da raiz dorsal, tem impacto clínico considerável, manifestando-se com dor, paresia e parestesias ${ }^{(43)}$.

O ligamento amarelo forma uma barreira anatômica para as raízes, dura-máter e gordura epidural, protegendo essas estruturas da compressão causada pelos tecidos circundantes; por isso, a sua preservação pode resultar em melhor prognóstico em relação à formação de fibrose epidural pós-discectomia ${ }^{(44)}$. 
Tem-se demonstrado associação entre fibrose e síndrome da falha da coluna operada (failed back) em $24 \%$ dos $\operatorname{casos}^{(45)}$.

As cirurgias de revisão para abordar esse problema elevam o risco de lesão neurológica e têm prognóstico desfavorável. O tratamento médico da fibrose é pouco efetivo. Múltiplas estratégias cirúrgicas e alguns dispositivos sintéticos foram usados para a sua prevenção, mas com resultados pouco satisfatórios. Por isso, se considera a prevenção ou inibição da formação da fibrose peridural como um dos mais importantes fatores prognósticos para o êxito da cirurgia.

Num estudo realizado pelo grupo de coluna do HUC, a avaliação favorável após no mínimo 10 anos de seguimento pós-operatório foi atribuída, entre outros fatores, à técnica microcirúrgica, que incluiu a preservação de ligamento amarelo (Figura 5). Esses resultados foram similares aos publicados por Ozer et $a^{(46)}$ e Askar et $a l^{(47)}$.

\section{RESULTADOS CLÍNICOS DA DISCECTOMIA}

Os resultados da discectomia convencional são variáveis. Em curto prazo, com dois anos de seguimento,

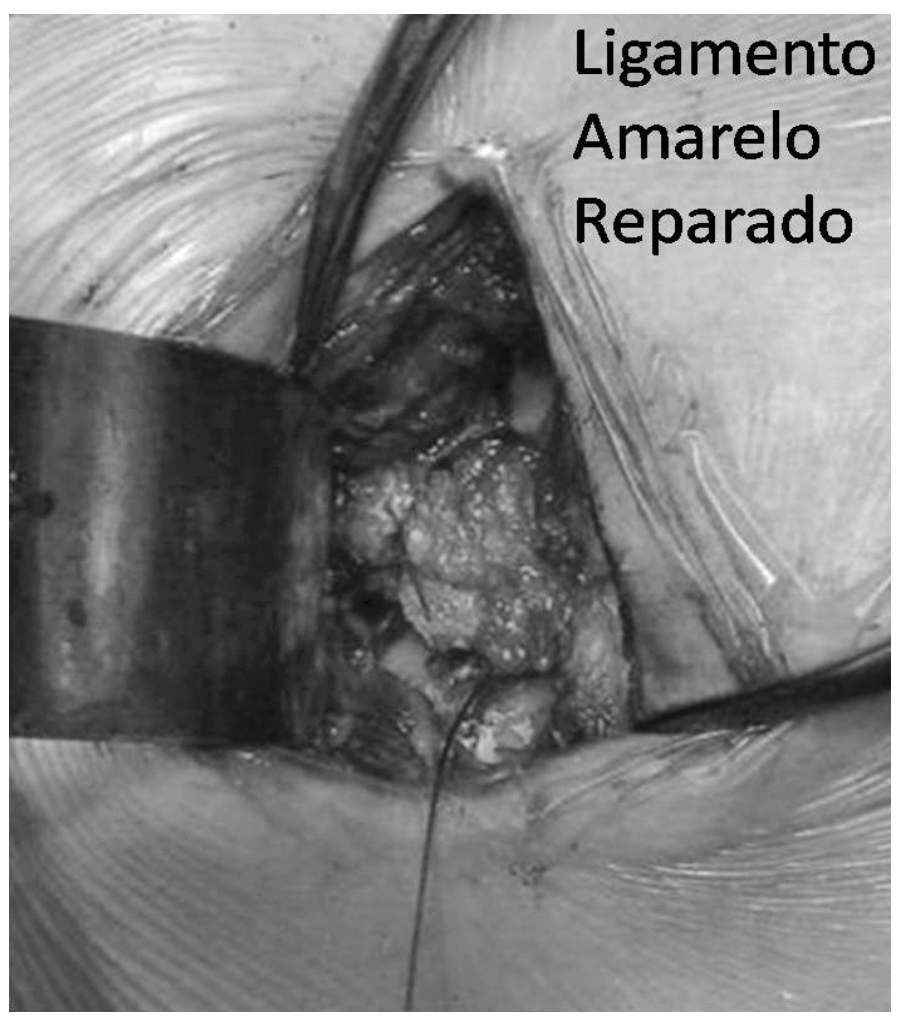

Figura 5 - Detalhe da técnica de sutura do ligamento amarelo encontram-se $90 \%$ de bons resultados; inversamente, em estudos em longo prazo - seis anos - encontraramse resultados insatisfatórios tão altos como $60 \%{ }^{(41,47)}$. Mas um estudo metodologicamente importante, cuja sigla é SPORT ${ }^{(48)}$, demonstrou que pacientes com hérnia discal lombar e radiculopatia, levados à cirurgia precoce, têm avaliação superior nos parâmetros considerados. Essa diferença se faz significativa após seis semanas de seguimento, alcança seu máximo beneficio aos seis meses e se mantém por quatro anos.

No estudo realizado pelo grupo de coluna do HUC, foi utilizada a escala analógica da dor e o índice de Oswestry, para avaliar os resultados após 10 anos em pacientes levados à discectomia, mostrando 87,9\% de bons resultados com respeito à dor irradiada.

Quando foram inclusos só pacientes com mais de 15 anos de seguimento, a frequência de bons resultados diminuiu, encontrando-se $21,9 \%$ de casos com incapacidade funcional moderada, o que está relacionado com a progressão da doença de base, a degeneração do disco intervertebral ${ }^{(34)}$.

\section{OUTROS MÉTODOS}

Nos últimos anos, tem havido uma orientação gradual para tratamentos menos invasivos para a hérnia discal, incluindo descompressão percutânea, descompressão com laser e, mais recentemente, descompressão usando um dispositivo de radiofrequência bipolar - também conhecido como nucleoplastia. Apesar de reduzirem a pressão intradiscal, seu real benefício ainda é controverso ${ }^{(49-51)}$.

\section{CONCLUSÃO E RECOMENDAÇÕES}

Hérnia de disco é uma patologia com um curso extremamente benigno.

O tratamento conservador é eficaz em $80 \%$ dos pacientes, dentro de quatro a seis semanas.

No caso de difícil controle da dor, bloqueio foraminal é a melhor opção.

A indicação cirúrgica deve ser proposta na falha do tratamento conservador, ou na progressão dos sintomas neurológicos.

Nesses casos, a microdiscectomia (lupa ou microscópio) com preservação do ligamento amarelo temse mostrado eficaz na prevenção de complicações, evitando fibrose peridural e reduzindo a recidiva sintomática. 


\section{REFERÊNCIAS}

1. Mixter WJ, Barr JS. Rupture of intervertebral disc with involvement of the spinal canal. N Engl J Med. 1934;211:210-4.

2. Love JG. Removal of intervertebral disc without laminectomy. Proc Staff Meet Mayo Clinic. 1939;14:800.

3. Sammes RE. Diagnosis of ruptured intervertebral disc without contrast myelography and comment upon recent experience with modified hemilaminectomy for their removal. Yale J Biol Med. 1939;11(5):433-5.

4. Spangfort EV. The lumbar disc herniation. A computer-aided analysis of 2504 operations. Acta Orthop Scand Suppl. 1972;142:1-95.

5. Garrido E. Lumbar disc herniation in the pediatric patient. Neurosurg Clin N Am. 1993;4(1):149-52.

6. Mayer HM, Mellerowicz H, Dihlmann SW. Endoscopic discectomy in pediatric and juvenile lumbar disc herniations. J Pediatr Orthop B. 1996;5(1):39-43.

7. Obukhov SK, Hankenson L, Manka M, Mawk JR. Multilevel lumbar disc herniation in 12-year old twins. Childs Nerv Syst. 1996;12(3):169-71.

8. Bortoletto A, Prata SD, Bonfim dos Santos G. Hérnia discal em crianças e adolescentes: relato de cinco casos. Rev Bras Ortop. 1998;33(10): 811-4.

9. Long DM, BenDebba M, Torgerson WS, Boyd RJ, Dawson EG. Hardy RW. Persistent back pain and sciatica in the United States: patient characteristics. J Spinal Disord. 1996;9(1):40-58.

10. Urban JP, Roberts S. Development and degeneration of the inter-vertebral discs. Mol Med Today. 1995;1(7):329-35.

11. Battie MC, Videman T, Gibbons LE, Fisher LD, Manninen H, Gill K. Volvo Award in Clinical Sciences. Determinants of lumbar disc degeneration. A study relating lifetime exposures and magnetic resonance findings in identical twins. Spine (Phila Pa 1976). 1995;20(24):2601-12.

12. Jones G, White C, Sambrook P, Eisman J. Allelic variation in the vitamin D receptor, lifestyle factors and lumbar spinal degenerative disease. Ann Rheum Dis.1998;57(2):94-9.

13. Videman T, Leppavuori J, Kaprio J, Battie MC, Gibbons LE, Peltonen L, et al. Intragenic polymorphisms of the vitamin $D$ receptor gene associated with intervertebral disc degeneration. Spine. 1998;23(23):2477-85.

14. Annunen S, Paassilta P, Lohiniva J, Perala M, Pihlayamaa T, Karppinen J, et al. An allele of COL9A2 associated with intervertebral disc disease. Science. 1999;285(5426):409-12.

15. Doege KJ, Coulter SN, Meek LM, Maslen K, Wood J. A human-specific polymorphism in the coding region of the aggrecan gene. Variable number of tandem repeats produce a range of core protein sizes in the general population. J Biol Chem. 1997;272(21):13974-9

16. Horton WE, Lethbridge-Cejku M, Hochberg MC, Balakir R, Precht P, Tobin JD, et al. An association between an aggrecan gene polymorphic allele and bilateral hand osteoarthritis in elderly white men: data from the Baltimore Longitudinal Study of Aging (BLSA). Osteoarthritis Cartilage. 1998;6(4):245-51.

17. Kawaguchi Y, Osada R, Kanamori M, Ishihara H, Ohmori K, Matsui H, et al. Association between an aggrecan gene polymorphism and lumbar disc degeneration. Spine (Phila Pa 1976). 1999;24(23):2456-60.

18. Kulisch SD, Ulstrom CL, Michael CJ. The tissue origin of low back pain and sciatica: a report of pain response to tissue stimulation during operations on the lumbar spine using local anesthesia. Orthop Clin North Am. 1991;22(2):181-7.

19. Vroomen P, de Krom M, Wilmink J, Kester A, Knottnerus J. Diagnostic value of history and physical examination in patients suspected of lumbosacral nerve root compression. J Neurol Neurosurg Psychiatry. 2002;72(5):630-4.

20. Olmarker K, Rydevik B, Holm S. Edema formation in spinal nerve roots induced by experimental, graded compression. An experimental study on the pig cauda equina with special reference to differences in effects between rapid and slow onset of compression. Spine (Phila Pa 1976). 1989;14(6):569-73.

21. Cornefjord M, Olmarker K, Rydevik R, Nordborg C. Mechanical and biochemical injury of spinal nerve roots: a morphological and neurophysiological study. Eur Spine J. 1996;5(3):187-92.

22. Howe JF, Loeser JD, Calvin WH. Mechanosensitivity of dorsal root ganglia and chronically injured axons: a physiological basis for the radicular pain of nerve root compression. Pain. 1977;3(1):25-41.

23. Fardon DF, Milette PC Nomenclature and classification of lumbar disc pathology. Recommendations of the Combined Task Forces of the North American Spine Society, American Society of Spine Radiology, and American Society of Neuroradiology. Spine (Phila Pa 1976). 2001;26(5):E93-E113.

24. Boss N. Disc herniation and radiculopathy. In: Boss N, Aebi M, editors. Spinal disorders: fundamentals of diagnosis and tratment. Berlin: Springer-Verlag Heidelberg; 2008.

25. Manchikanti L, Boswell MV, Singh V, Benyamin RM, Fellows B, Abdi S, et al. Comprehensive evidence-based guidelines for interventional techniques in the management of chronic spinal pain. Pain Physician. 2009;12(4):699-802.

26. Autio RA, Karppinen J, Kurunlahti M, Haapea M, Vanharanta $H$, Tervonen O. Effect of periradicular methylprednisolone on spontaneous resorption of intervertebral disc herniations. Spine (Phila Pa 1976). 2004;29(15):1601-7.
27. Riew KD, Park JB, Cho YS, Gilula L, Patel A, Lenke LG, Bridwell KH. Nerve root blocks in the treatment of lumbar radicular pain. A minimum five-year follow-up. J Bone Joint Surg Am. 2006;88(8):1722-5.

28. Wang JC, Lin E, Brodke DS, Youssef JA.. Epidural injections for the treatment of symptomatic lumbar herniated discs. J Spinal Disord Tech. 2002;15(4):269-72.

29. Vialle E, Vialle LR, Carvalho LF, Godinho P. Bloqueio radicular lombar via transforaminal no manejo da hérnia discal lombar, Grupo de Cirurgia da Coluna, Hospital Universitário Cajuru, PUC-PR. In: XII Anais do Congresso Brasileiro da Coluna, Foz do Iguaçu, PR, 2007.

30. Buenaventura RM, Datta S, Abdi S, Smith HS. Sistematic review of therapeutic lumbar transforaminal epidural steroids injections. Pain Physician. 2009;12(1):233-51

31. Khadilkar A, Odebiyi DO, Brosseau L, Wells GA. Transcutaneous Electrical Nerve Stimulation (TENS) versus placebo for chronic low-back pain. Cochrane Database Syst Rev. 2008;(4): CD003008.

32. Van den Hout WB, Peul WC, Koes BW, Brand R, Kievit J, Thomeer RT; Leiden-The Hague Spine Intervention Prognostic Study Group. Prolonged conservative care versus early surgery in patients with sciatica from lumbar disc herniation: cost utility analysis alongside a randomised controlled trial. BMJ. 2008;336(7657):1351-4.

33. Peul WC, Van den Hout WB, Brand R, Thomeer RT, Koes BW; Leiden-The Hague Spine Intervention Prognostic Study Group. Prolonged conservative care versus early surgery in patients with sciatica caused by lumbar disc herniation: two years results of a randomized controlled trial. BMJ. 2008;336(7657):1355-8.

34. Vialle LR, Vialle E, Martins Filho DE. Seguimento de longo prazo após cirurgia de hérnia de disco lombar. Grupo de Cirurgia da Coluna, Hospital Universitário Cajuru, PUCPR. In: XII Anais do Congresso Brasileiro da Coluna, Foz do Iguaçu, PR, 2007.

35. Watters WC 3rd, McGirt MJ. An evidence-based review of the literature on the consequences of conservative versus aggressive discectomy for the treatment of primary disc herniation with radiculopathy. Spine J. 20099(3):240-57.

36. Ruetten S, Komp M, Merk H, Godolias G. Full-endoscopic interlaminar and transforaminal lumbar discectomy versus conventional microsurgical technique: a prospective, randomized, controlled study. Spine (Phila Pa 1976). 2008;33(9):931-9.

37. Arts MP, Brand R, van den Akker ME, Koes BW, Bartels RH, Peul WC, et al. Tubular diskectomy vs conventional microdiskectomy for sciatica: a randomized controlled trial. JAMA. 2009;302(2):149-58.

38. Thomé C, Barth M, Scharf J, Schmiedek P. Outcome after lumbar sequestrectomy compared with microdiscectomy: a prospective randomized study. J Neurosurg Spine. 2005;2(3):271-8.

39. Carragee EJ, Spinnickie AO, Alamin TF, Paragioudakis S. A prospective controlled study of limited versus subtotal posterior discectomy: short-term outcomes in patients with herniated lumbar intervertebral discs and large posterior anular defect. Spine (Phila Pa 1976). 2006;31(6):653-7.

40. Yorimitsu E, Chiba K, Toyama Y, Hirabayashi K. Long-term outcomes of standard discectomy for lumbar disc herniation: a follow-up study of more than 10 years. Spine (Phila Pa 1976). 2001;26(6):652-7.

41. Loupasis GA, Stamos K, Katonis PG, Sapkas G, Korres DS, Hartofilakidis G. Seven- to 20-year outcome of lumbar discectomy. Spine (Phila Pa 1976). 1999;24(22):2313-7.

42. Wolfram-Gabel R, Beaujeux R, Fabre M, Kehrli P, Dietemann JL, Bourjat P. Histologic characteristics of posterior lumbar epidural fatty tissue. J Neuroradiol. 1996;23(1):19-25.

43. Olmarker K, Rydevik B. Pathophysiology of sciatica. Orthop Clin North Am. 1991;22(2):223-34

44. Masopust V, Häckel M, Netuka D, Bradác O, Rokyta R, Vrabec M. Postoperative epidural fibrosis. Clin J Pain. 2009;25(7):600-6.

45. Ross JS, Robertson JT, Frederickson RC, Petrie JL, Obuchowski N, Modic MT, et al. Association between peridural scar and recurrent radicular pain after lumbar discectomy: magnetic resonance evaluation. ADCON-L European Study Group Neurosurgery. 1996;38(4):855-61.

46. Ozer AF, Oktenoglu T, Sasani M, Bozkus H, Canbulat N, Karaarslan E, et al. Preserving the ligamentum flavum in lumbar discectomy: a new technique that prevents scar tissue formation in the first 6 months postsurgery. Neurosurgery. 2006:59(1 Suppl 1):ONS126-33.

47. Askar Z, Wardlaw D, Choudhary S, Rege A. A ligamentum flavum-preserving approach to the lumbar spinal canal. Spine (Phila Pa 1976). 2003;28(19):E385-90.

48. Weinstein JN, Lurie JD, Tosteson TD, Tosteson AN, Blood EA, Abdu WA. Surgical versus nonoperative treatment for lumbar disc herniation: four-year results for the Spine Patient Outcomes Research Trial (SPORT). Spine (Phila Pa 1976). 2008;33(25):2789-800.

49. Manchikanti L, Derby R, Benyamin R, Standiford H, Hirsh JA. A systematic review of mechanical lumbar disc decompression with nucleoplasty. Pain Physician. 2009;12(3):561-72.

50. Singh V, Benyamin RM, Datta S, Falco F, Standiford H, Hirsh JA, Manchikanti L. Systematic review of percutaneus lumbar mechanical disc decompression utilizing Dekompressor. Pain Physician. 2009;12(3):589-99.

51. Singh V, Manchikanti L, Benyamin R, Standiford H, Hirsh JA. Percutaneus lumbar laser disc decompression: a systematic review of current evidence. Pain Physician. 2009;12(3):573-88. 